Meta

Journal des tradlucteurs

Translators' Journal

\title{
Sémantique syntagmatique et traduction : des notions qui sont aussi des outils
}

\section{Jean-Claude Choul}

Volume 31, numéro 2, juin 1986

URI : https://id.erudit.org/iderudit/002239ar

DOI : https://doi.org/10.7202/002239ar

Aller au sommaire du numéro

Éditeur(s)

Les Presses de l'Université de Montréal

ISSN

0026-0452 (imprimé)

1492-1421 (numérique)

Découvrir la revue

Citer cet article

Choul, J.-C. (1986). Sémantique syntagmatique et traduction : des notions qui sont aussi des outils. Meta, 31(2), 159-164. https://doi.org/10.7202/002239ar d'utilisation que vous pouvez consulter en ligne.

https://apropos.erudit.org/fr/usagers/politique-dutilisation/ 


\section{SÉMANTIQUE SYNTAGMATIQUE ET TRADUCTION : DES NOTIONS QUI SONT AUSSI DES OUTILS}

JEAN-ClaUde Choul UNIVERSITÉ DE LA SASKATCHEWAN

La plupart des théories sémantiques (et la sémantique elle-même, même dans sa phase comparatiste) se sont constituées en dehors des applications, sans doute par souci de généralité. En fait, cette généralité peut être transposée dans la traduction, mais demande une synthèse d'éléments apparemment hétéroclites.

L'idéal serait de disposer d'une théorie sémantique qui réponde aux besoins de la traduction, et principalement du traducteur, dans l'exercice de sa profession. C'est-àdire d'une théorie du sens qui permette de traduire et d'expliquer pourquoi et comment se traduisent tous les énoncés possibles d'une langue quels que soient les écarts manifestés.

Une théorie suffisamment générale donc, donnant au traducteur les moyens de cerner les types de phénomènes les plus divers et de les rendre sans recourir à des solutions ad hoc.

On pourrait par exemple partir d'une observation du travail du traducteur pour construire le cadre théorique nécessaire à l'explication des mécanismes en jeu. Il s'agirait là d'une macro-théorie, s'appuyant sur une théorie de la compréhension du langage. Une telle théorie pourrait par exemple se constituer autour de dispositifs sémiotiques : les univers sollicités au cours de l'élaboration d'un texte B à partir d'un texte A.

Une théorie de ce type me semble possible si avant de considérer le traducteur comme intermédiaire dans un schéma de communication on le présente comme récepteur, mais récepteur actif. Quelqu'un qui, devant une séquence de signaux, transforme ces signaux en signification : un lecteur.

J'ai $\mathrm{pu}$, au cours d'une recherche antérieure, esquisser un modèle de réceptionperformance qui cherche à rendre compte des processus caractérisant la lecture. Ce modèle macro-théorique déjà fondé sur le transcodage ne présente aucune difficulté d'adaptation à la traduction. Le transcodage ajouté est évidemment interlingual, et s'insère après le transcodage intralingual.

Ce modèle sera sous-jacent à mon exposé, car une autre théorie de la traduction est possible, à l'intérieur de ce premier modèle : une micro-théorie portant sur la compréhension des réalisations partielles du langage, fondée sur les mécanismes intraénoncés. Par réalisations partielles, je pense surtout aux matériaux préfabriqués comme nose cone, pincer attack, catwalk, bulk carrier, coke machine, etc., dont la théorie devra décrire les relations sémantiques avant de s'en rapporter à la désignation. C'est-à-dire au lieu de procéder par simple substitution, fondée sur le principe du "en anglais ça s'appelle - en français ça s'appelle ».

C'est en cela qu'une telle théorie est dite syntagmatique. Et si elle est sémantique, c'est qu'elle porte sur le sens. Cette micro-théorie n'est pas indépendante du modèle global. En fait, si elle présente quelque validité, elle comportera autant de "mécanismes" 
qu'il y a d'opérations dans la première phase du modèle de compréhension. Elle se présenterait ainsi en miroir de la macro-théorie.

Le terme de mécanisme ne doit pas nous abuser : il s'agit plutôt de phénomènes qui se prêtent à l'observation. Une première et triple distinction terminologique va nous permettre de mettre en place les composantes fondamentales de la micro-théorie. Nous examinerons ensuite les notions essentielles de l'appareil théorique.

Sens

Le sens tout d'abord se situe entre le sémantisme et la signification : c'est-à-dire qu'il s'agit d'une étape dans un procès dont la signification est le terme. Cette étape correspond à l'insertion en contexte linguistique. On sait que le contexte précise et fixe le sens d'un mot ou lexème. Il serait plus juste de dire que la contextualisation transforme le sémantisme en sens, à l'égal de la mise en situation qui transforme le sens en signification. Le sémantisme de nose est assez étendu, mais le sens de nose dans the nose of an aircraft ne conserve qu'une seule possibilité correspondant à une traduction quasi instantanée : le nez d'un avion.

Le sens est tenu à une certaine constance en vertu des présomptions propres à la communication, mais la signification est dotée d'une grande variabilité : je vais faire un tour de moto peut très bien devenir je vais me suicider.

\section{Sémantisme}

Le sémantisme est ainsi non seulement préalable à l'analyse, mais également à la contextualisation. On peut le définir de façon opératoire en. le présentant comme l'ensemble des entrées possibles des dictionnaires unilingues pour une forme lexicale donnée, qu'il s'agisse de polysémèmes ou d'homonymes. Le sémantisme global de load (charge) peut être illustré par les exemples suivants qui marquent le rapport qui existe entre polysémie et contexte :
load of wood
load on a heating system
truck load
working load of an engine
load on a beam
load of a power station
load of a washing machine
You've taken a load off my mind.

\section{Sémantisation}

On peut aussi rattacher le sémantisme à la sémantisation qui se produit à la lecture d'une unité non mémorisée (inconnue) et qui dote cette unité de valeurs et de propriétés.

Ainsi vigneaux claies dans la phrase :

Plus tard, il faudrait sortir ce poisson et le ranger sur les vigneaux claies où le soleil se chargerait de le sécher. (Y. Thériault, la Passe-au-crachin.)

le sémantisme consisterait en des propositions du type : /objet sur lequel on dispose le poisson pour le faire sécher au soleil (à l'air libre)/. Il s'agit ici d'une reconstruction, qui ne doit rien à la consultation d'un dictionnaire. Bien sûr, claie peut apporter /treillis à claire-voie/, et à la consultation vigneaux pourrait recevoir la définition suivante : /tertre surmonté d'une treille/. On peut aussi vérifier la sémantisation en substituant l'expression quelque chose aux éléments étudiés.

Toutefois, si le phénomène de la sémantisation est intéressant à signaler, il comporte en corollaire une mise en garde ; le contexte suivant ne permet pas de sémantiser 
voilier de façon satisfaisante et ne remplace pas la compétence lexicale spécialisée qui nous est fournie par le Petit Larousse.

La mouette est un voilier très résistant mais peu rapide : elle peut planer pendant des heures, profitant des courants aériens et gagnant souvent assez de vitesse pour aller à contre vent.

Si lorsqu'il s'agit d'un oiseau, voilier veut dire « dont le vol est étendu » (ce qui est le cas selon le Petit Larousse), on voit que les vertus du contexte dans la sémantisation ont des limites. On doit donc discipliner la sémantisation.

\section{Redondance}

Si la sémantisation est une opération qui a pour objet d'assigner une valeur sémantique à un "blanc sémantique " dans la cohérence présumée d'un énoncé, elle s'exerce sur un matériau que la notion de redondance permet de cerner.

Celle-ci est diversement connue, mais une illustration de la redondance telle qu'elle est entendue ici nous est donnée par l'exemple suivant :

He says open fires stultify mental processes. (Rex Stout, Gambit.)

Il suffit de substituer l'une des acceptions du Longman's à la forme lexicale qui nous intéresse : stultify :

Open fires make stupid or dull (in mind) mental processes.

où l'on voit que mind (et même stupid) est repris par mental (of the mind). C'est ce que Bernard Pottier appelle isosémie : il faut que les lexèmes associés en contexte proche aient au moins un sème en commun.

La sémantisation (en cas d'absence de sème partagé) va donc consister à fabriquer de la redondance, même à tort, tant est forte la présomption de cohérence du discours humain.

La sémantisation est un phénomène important dans la traduction en raison de l'inertie propre au traducteur (peu enclin à soulever le dictionnaire).

On peut donc délimiter la redondance sémantique par cette observation : tout contexte doit posséder des traits qui appartiennent à la définition des lexèmes cooccurrents, mais on se souviendra que ces traits sont mobiles, et qu'ils peuvent faire l'objet d'un transfert, qui est l'équivalent syntagmatique de la sémantisation par corrélation avec une forme absente de l'énoncé.

La redondance comme congruence sémantique (isosémie) est une notion plus souple que les relations de sélection des grammaires chomskyennes qui se présentent comme règles - et dès qu'il y a règles, les exceptions ne manquent pas, ni les contreexemples. Il suffit de signaler $d r i n k$, verbe exigeant un objet possédant le trait /liquide/, mais que fera-t-on de drink someone under the table?

\section{Transfert}

Le transfert peut être illustré comme la recherche de la motivation d'une cooccurrence. Ainsi, l'apparition d'un assemblage du genre de grue métaphysique déclenche la recherche d'un sémantisme partagé, c'est-à-dire de ce qui motive la collocation de lexèmes ne présentant à première vue aucune redondance, c'est-à-dire aucun collocable commun. De fait, ce n'est que par le biais d'un paramètre comme /soulever un problème/ que l'on peut justifier l'expression.

Le transfert serait donc la première manifestation syntagmatique. La seconde, nous l'avons entrevue avec stultify, lorsque nous avons substitué l'une des acceptions, 
que nous avons choisi celle qui présentait une redondance (le transfert était donc déjà réalisé). Il s'agit de la sélection. Le phénomène est assez connu, puisqu'il figure dans le Dictionnaire de didactique des langues de Galisson et Coste.

Prendre un café, prendre un autobus, prendre quelque chose à quelqu'un, prendre quelqu'un pour un homme habile, distinguent autant de sens différents de prendre : absorber, faire usage d'un moyen de transport, voler, considérer.

\section{Sélection}

En fait, la compatibilité sémantique tient comme nous vẹnons de le voir au partage des sèmes (à leur redondance), ce qui se représente mieux si l'on admet que les sèmes communs sont actualisés par les collocables communs. S'asseoir dans un fauteuil est redondant dans la mesure où le sème /s'asseoir/ apparaît dans le sémiogramme (description sémique) de fauteuil. Pour le traducteur, cette vérification de la collocabilité se fait de manière privilégiée dans la comparaison des phrases-exemples des dictionnaires aux contextes qu'il doit traduire.

Le contrôle de la redondance permet au traducteur de s'assurer que la sélection opérée est celle qui convient. Il évite par exemple de traduire manufacture par manufacture, dont la distribution est restreinte en français. Il en va de même pour l'équivalence supposée entre operate et opérer.

La sélection écarte ou réduit la polysémie : elle est caractéristique de la syntagmation, que Benveniste présente comme appauvrissement sémantique (perte de valeur du signe). C'est la sélection qu'illustrent les exemples de Nida avec run lorsqu'il introduit les marques sémantiques : semotaxis. Voici certains de ses exemples, dont la traduction met bien en évidence la sélection qu'opère la syntagmation dans le sémantisme :

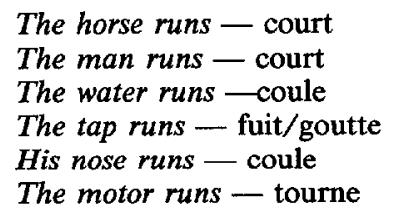

Certains dictionnaires de langue utilisent, et de tout temps les dictionnaires bilingues ont utilisé, les constructions (intransitives, transitives, directes, indirectes) pour distinguer les acceptions.

\section{Sémiotaxie}

L'apport de Guiraud à propos de la nature syntaxique du sens met à notre disposition une notion très utile : la sémiotaxie. C'est-à-dire un assemblage sémantique, et cette notion dynamique va regrouper le transfert et la sélection.

Voici quelques éléments possibles de la sémiotaxie de compter :

compter 0 (faire un calcul) savoir compter

compter 0 (être important) la gloire compte

compter sur qqn (faire fond/se fier à) avec qqn (tenir compte de)

compter qqn/qqch pour rien (ne pas faire cas)

Cependant la sémiotaxie ne sera complète que si on lui ajoute le troisème phénomène que l'on peut observer dans la syntagmation, phénomène étroitement lié aux deux précédents, et conséquence immédiate de la sélection.

Il s'agit de la spécialisation sémantique. 


\section{Spécialisation}

La meilleure illustration de la spécialisation, pour le traducteur, se trouve dans les dictionnaires ; il s'agit bien sûr de la sous-entrée et plus particulièrement de l'indication codée : spécialement. À l'entrée solution, par exemple, on trouvera solution de continuité. À chaise on trouvera une variété de chaises, de toute évidence, mais chaise électrique constitue l'extrême dans la spécialisation.

Il est bon de noter que la spécialisation peut s'exercer sur un lexème autant que sur un syntagme, mais il suffit de reconstruire le syntagme pour bien faire ressortir la spécialisation et montrer qu'elle est le résultat de la syntagmatisation. Voici quelques cas, avec lunette :

$\begin{array}{ll}\text { lunette astronomique } & \text { lunette des w.-c. } \\ \text { lunette d'approche } & \text { lunette de l'oiseau } \\ \text { lunette de pointage (de tir) } & \text { lunette du toit } \\ \text { lunettes d'écaille } & \text { lunette d'un fort } \\ \text { lunettes de soleil } & \text { lunette de la voiture } \\ \text { lunettes de plongeur } & \text { lunette d'étambot } \\ \text { lunettes noires } & \text { lunette de la guillotine } \\ \text { lunette de la montre } & \text { lunette d'un tour }\end{array}$

lunette pyrométrique

On peut faire correspondre la spécialisation à l'inscription du domaine ou de la situation dans le syntagme, c'est-à-dire que l'information extralinguistique n'est pas exclue d'une syntagmatique.

À la spécialisation correspond une notion du macro-modèle : la paraphrase. Celleci est bien connue des didacticiens, puisque son exercice est condition de l'apprentissage du sens.

\section{Paraphrase}

La paraphrase permet de vérifier si oui ou non il y a spécialisation, et si le degré est suffisant pour qu'il y ait suspension sémique et début de paramétrisation (lexicalisation partielle ou totale du syntagme).

On remarque une spécialisation croissante dans les séries :

bulle d'air (globule)

bulle papale (décret)

tache de graisse (marque salissante)

chambre à bulles (instrument)

tache de rousseur (marque naturelle)

La spécialisation se marque par une paraphrase de moins en moins transparente, ce qui nécessite un contexte plus étendu pour réduire la polysémie, donc l'apparition de formes lexicales complexes.

Voici quelques exemples avec draw :

draw water (obtain)

draw tea (extract by infusion)

draw flies (attract)

draw on (approach - evening)

draw off (remove, withdraw - gloves/liquid)

Il faut noter que la traduction française se fait dans ce cas sur la paraphrase, et non sur le terme d'origine.

Il est possible qu'il y ait des limites à l'approche paraphrastique. Selon J.-C. Milner, seuls les processus peuvent se paraphraser et non les objets. On rejoint alors les problèmes de la définition : de quoi s'agit-il sinon d'une paraphrase faisant apparaître les sèmes pertinents?

L'extension du contexte combinée à l'opacification (occultation du sens) va donner ce que l'on peut appeler des paramètres. 


\section{Paramètre}

La notion de paramètre est empruntée à l'écrivain Jean Paulhan qui la proposait pour rendre compte des " figures " que sont les énoncés cohésifs, contextes constants en discours, de la locution au proverbe. Elle est parallèle à la lexie complexe et permet de souligner l'exploitation possible des formes dites figées, puisque l'interprétation des énoncés déviants se fera à l'aide de l'ensemble constant. C'est le cas des jeux de mots, comme la publicité de Philips sur les fibres optiques avec light fantastic (trip the light fantastic = danser), ou les titres : The Father Hunt (man hunt = chasse à l'homme), Plot if Yourself (do it yourself), etc.

La notion de paramètre permet en outre de ne pas séparer les faits phraséologiques des faits syntagmatiques, et de marquer une continuité de la collocabilité (comme présyntagmation) aux locutions (blocage graduel des substitutions, reprises, coordination).

Ce n'est en effet que la stabilisation, subséquente à la spécialisation et extralinguistique, qui permet de marquer une différence entre les lexèmes qui se réunissent souvent et ceux qui apparaissent obligatoirement ensemble.

Il est difficile, par exemple, de déterminer si donner son opinion est phraséologique ou syntagmatique ; tout ce que l'on peut dire c'est que opinion a le sens d'avis, tandis que braver l'opinion consiste à affronter le qu'en dira-t-on et qu'un journal d'opinion est un journal de tendance et qu'avoir une bonne opinion de soi-même c'est être content de soi.

On voit que sélection, paraphrase et paramètre sont liés.

Pour le traducteur, l'observation de ces phénomènes est fondamentale s'il veut faire autre chose que transposer une syntaxe et des lexèmes d'une langue dans l'autre.

La sémantique syntagmatique ou l'étude des phénomènes sémantiques observables dans la syntagmation n'est pas une théorie de la traduction, mais elle met en place des notions qui dans la perspective du transcodage peuvent devenir des outils.

Ces observations sont toujours en cours et la recherche envisage maintenant la mise au point de modules syntagmatiques ou sémiotaxiques exploitant les rapports entre le sens (acception ou paraphrase) et la construction, en partant d'un terme pour constituer les paramètres liés à ce terme ; cette entreprise devrait permettre d'approfondir la connaissance du syntagme.

\section{RÉFÉRENCES}

BENVENISTE, E. (1976) : Problèmes de linguistique générale II, Paris, Gallimard.

CHOUL, J.-C. (1979) : le Sens de la lecture, contributions aux recherches sémantiques et sémiotiques, thèse de doctorat de $3^{\mathrm{e}}$ cycle, inédite, Paris, École des hautes études en sciences sociales.

CHOUL, J.-C. (1979) : " La méthode du discours : quelques outils ", META 24-3, Montréal, PUM.

CHOUL, J.-C. (1980) : "Un modèle sémiotique de la traduction", Revue canadienne des sciences de l'information, Montréal, ACSI.

CHOUL, J.-C. (à paraître) : "Sémantique et traduction : exercices".

GUIRAUD, P. (1970) : Structures étymologiques du lexique français, Paris, Larousse.

MILNER, J.-C. (1978) : De la syntaxe à l'interprétation : quantités, insultes, exclamations, Paris, Seuil.

NIDA, E.A. et C.R. TABER (1969) : The Theory and Practice of Translation, Leyde, E.J. Brill.

PAULHAN, J. (1966) : Traité des figures, in J. Paulhan, OEuvres complètes, Paris, Cercle du livre précieux, tome II, pp. 195-237.

POTTIER, B. (1974) : Linguistique générale, théorie et description, Paris, Klincksieck. 